\title{
The present and future of serum diagnostic tests for testicular germ cell tumours
}

\section{Matthew J. Murray ${ }^{1,2}$, Robert A. Huddart ${ }^{3,4}$ and Nicholas Coleman ${ }^{1,5}$}

${ }^{1}$ Department of Pathology, University of Cambridge, Tennis Court Road, Cambridge, CB2 1QP, UK.

${ }^{2}$ Department of Paediatric Haematology and Oncology, Cambridge University Hospitals NHS Foundation Trust, Cambridge, CB2 0QQ, UK.

${ }^{3}$ The Institute of Cancer Research, Downs Road, Sutton, Surrey, SM2 5PT, UK.

${ }^{4}$ The Royal Marsden NHS Foundation Trust, Downs Road, Sutton, Surrey, SM2 5PT, UK.

${ }^{5}$ Department of Histopathology, Cambridge University Hospitals NHS Foundation Trust, Cambridge, CB2 0QQ, UK.

Correspondence to M.J.M. and N.C.

mjm16@cam.ac.uk

nc109@cam.ac.uk

\begin{abstract}
Testicular germ cell tumours (GCTs) are the most common malignancy occurring in young adult men and the incidence of these tumours is increasing. Current research priorities in this field include improving overall survival for patients classified as being 'poor-risk' and reducing late effects of treatment for patients classified as 'goodrisk'. Testicular GCTs are broadly classified into seminomas and nonseminomatous GCTs (NSGCTs). The conventional serum protein tumour markers $\alpha$-fetoprotein (AFP),
\end{abstract}


human chorionic gonadotrophin (HCG) and lactate dehydrogenase (LDH) show some utility in the management of testicular malignant GCT. However, AFP and HCG display limited sensitivity and specificity, being indicative of yolk sac tumour (AFP) and choriocarcinoma or syncytiotrophoblast (HCG) subtypes. Furthermore, LDH is a very nonspecific biomarker. Consequently, seminomas and NSGCTs comprising a pure embryonal carcinoma subtype are generally negative for these conventional markers. As a result, novel universal biomarkers for testicular malignant GCTs are required. MicroRNAs are short, non-protein-coding RNAs that show much general promise as biomarkers. MicroRNAs from two 'clusters', miR-371-373 and miR-302-367, are overexpressed in all malignant GCTs, regardless of age (adult or paediatric), site (gonadal or extragonadal) and subtype (seminomas, yolk sac tumours or embryonal carcinomas). A panel of four circulating microRNAs from these two clusters (miR-371a-3p, miR-372-3p, miR-373-3p and miR-367-3p) is highly sensitive and specific for the diagnosis of malignant GCT, including seminoma and embryonal carcinoma. In the future, circulating microRNAs might be useful in diagnosis, disease monitoring and prognostication of testicular malignant GCTs, which might also reduce reliance on serial CT scanning. For translation into clinical practice, important practical considerations now need addressing.

\section{Key points}

- The conventional serum protein tumour markers $\alpha$-fetoprotein (AFP), human chorionic gonadotrophin (HCG) and lactate dehydrogenase (LDH) show utility in the management of testicular malignant germ cell tumours (GCTs) 
- AFP and HCG show limited sensitivity and specificity for all malignant GCTs, being representative of yolk sac tumour and choriocarcinoma or synctiotrophoblast subtypes, respectively; LDH is a very nonspecific biomarker

- Novel universal biomarkers for testicular malignant GCTs are required, particularly for seminoma and embryonal carcinoma subtypes that are typically negative for conventional markers

- MicroRNAs are short, non-protein-coding RNAs that show much promise as universal markers in malignant GCTs

- Individual microRNAs from two microRNA clusters, miR-371-373 and miR302-367, are overexpressed in all malignant GCTs, regardless of patient age, tumour site and subtype

- A panel of four circulating microRNAs from these two clusters (miR-371a-3p, miR-372-3p, miR-373-3p and miR-367-3p) is highly sensitive and specific for the diagnosis of malignant GCT, including seminoma and embryonal carcinoma subtypes

- Practical considerations need to be addressed to standardize the translation of circulating microRNA studies from a research tool to a routine clinical test

Testicular germ cell tumours (GCTs) are the most common malignancy occurring in young adult males and the incidence of these tumours is increasing over time ${ }^{1}$. Current research aims include increasing overall survival for patients deemed to have poor risk disease and improving quality of survival for patients with low-stage and/or good-risk 
disease. Testicular GCTs are broadly classified into seminoma (a malignant subtype) and nonseminomatous GCTs (NSGCTs). The latter group include the malignant subtypes yolk sac tumour (YST), embryonal and choriocarcinoma (CHC), as well as teratoma. Teratomas in prepubertal patients are generally considered benign, but postpubertal cases might display malignant potential ${ }^{2}$. GCTs that contain more than one subtype are known as mixed malignant GCTs; those with both seminomatous and nonseminomatous components are classified as combined NSGCTs ${ }^{3}$.

The histological classification and clinical staging of testicular GCTs guide subsequent treatment decisions. Stage I testicular GCTs (that is, those confined to the testes) are initially treated with orchidectomy, although management after orchidectomy varies and can be dictated by clinician and/or national preference ${ }^{4}$ and/or known risk factors ${ }^{5-10}$. Chemotherapy is routinely administered for patients with stage II-IV testicular GCTs according to International Germ Cell Cancer Collaboration (IGCCC) prognostic criteria $^{11}$.

The conventional serum protein biomarkers $\alpha$-fetoprotein (AFP) and human chorionic gonadotropin (HCG) are used to assist in the diagnosis and follow-up assessment of GCTs, but their use is generally restricted to tumours containing the relevant malignant subtypes YST and CHC, respectively ${ }^{12}$ (Table 1). Consequently, only $~ 60 \%$ of patients with testicular malignant GCTs test positive for these markers at diagnosis ${ }^{13}$. Serum lactate dehydrogenase (LDH) levels are also used at diagnosis to assist with treatment decisions in patients with metastatic NSGCTs but this marker lacks sufficient specificity 
to be useful in diagnosis or monitoring ${ }^{3}$ (Table 2). Current priorities in GCT research include the identification of universal circulating biomarkers of malignant disease, which might assist timely diagnosis and reduce the need for repeated cross-sectional CT imaging in follow-up monitoring, along with the associated radiation burden and risk of second cancer development ${ }^{14}$. One recent study, for example, showed that only $3 \%$ of patients relapsing following a diagnosis of stage I seminoma were identified using conventional serum tumour markers (CTMs), but that figure was $87 \%$ for CT imaging ${ }^{9}$. Other important issues that remain to be fully addressed include the most appropriate management of stage I testicular GCTs, particularly NSGCTs (active surveillance versus adjuvant chemotherapy), and the early identification of patients who are destined to have poor clinical outcomes within the overall IGCCC poor-risk disease cohort ${ }^{11}$. These issues are important, as many concerns exist regarding the substantial long-term sequelae of conventional chemotherapy treatment ${ }^{15-21}$, which include pulmonary fibrosis (with bleomycin), nephrotoxicity and/or ototoxicity (with cisplatin), neuropathy (with cisplatin and taxol agents) ${ }^{20}$. Particular concerns also exist about the increased incidence of cardiovascular disease ${ }^{17,} 18$ and second cancer $^{15,21}$ associated with conventional chemotherapy. Minimizing these risks through improved patient stratification is important; novel circulating biomarkers might assist with this challenge.

This Review provides a comprehensive overview of the current clinical value of serum diagnostic testing for testicular GCTs and discusses how the issues highlighted above could be addressed. It covers the utility of the traditional markers AFP, HCG and LDH in the diagnostic and prognostic management of this disease. In addition, it provides insights into the rapidly developing area of quantification of specific circulating short non- 
protein-coding RNAs termed microRNAs in GCTs, and discusses potential clinical applications of these markers. Practical aspects that must be considered before such microRNA testing can be incorporated into future routine clinical practice are also highlighted. The ultimate aims of developing circulating microRNA testing alongside conventional tumour marker testing are threefold. Firstly, to rationally reduce or omit the use of CT scans in follow-up for patients whose disease is usually negative for conventional markers (predominantly seminoma subtype) at diagnosis. Secondly, to improve quality of patient survival, through the rational reduction and/or omission of chemotherapy, along with its concomitant late effects, for patients with stage I disease, who are predicted to have excellent outcomes. Thirdly, to improve overall survival for those patients with poor-risk disease, through identification of a robust prognostic signature.

\section{[H1] AFP, HCG and LDH in testicular GCT}

The serum protein markers AFP, HCG and LDH have all shown utility for the management of testicular GCTs and are currently widely used in clinical practice ${ }^{3}$.

\section{[H2] Physiology, background and limitations}

AFP is a glycoprotein comprising a carbohydrate moiety attached to an 600 amino acid $\alpha$-globulin molecule ${ }^{22}$. In fetal development, AFP is produced by the yolk sac and then predominantly the liver, with a minor contribution from the gastrointestinal tract in later gestation. ${ }^{23}$ Postnatally, AFP is gradually replaced as the major circulating protein by albumin, which is exclusively derived from the $\operatorname{liver}^{22}$. In clinical practice, AFP levels are 
determined using a quantitative automated chemiluminescent sandwich enzyme immunoassay ${ }^{24}$, and measured against known AFP standards according to WHO International Standard $72 / 225^{22}$. The half-life of AFP in the bloodstream is usually $\sim 5-7$ days ${ }^{3,22,25}$ and levels are measured as $\mathrm{ng} / \mathrm{ml}$ or $\mathrm{kU} / \mathrm{l}$, with normal values being $<12 \mathrm{ng} / \mathrm{ml}(<10 \mathrm{kU} / \mathrm{l}$; conversion $1 \mathrm{ng} / \mathrm{ml}=0.84 \mathrm{kU} / \mathrm{l}){ }^{22}$. The features of AFP production during development are pertinent to the elevations observed in the serum of some patients with GCTs. Raised serum AFP levels are predominantly observed in GCTs containing YST components, although moderately elevated values can also occur in some embryonal carcinoma and immature teratoma lesions ${ }^{22}$ (Table 1). In pure immature teratoma, serum AFP can be raised owing to the presence of immature liver and/or gastrointestinal tissues within the lesion ${ }^{22}$.

AFP values are typically elevated in patients with hepatocellular carcinoma ${ }^{3}$, in patients with other uncomplicated chronic liver disease, ${ }^{26}$ those who have undergone gastrointestinal tract and/or hepatic surgery and in patients with certain conditions such as hereditary ataxia telangiectasia ${ }^{27}$. Infrequent reports of elevated AFP levels have also been described in gastrointestinal malignancies ${ }^{3,22}$ (including gastric, pancreatic and colon carcinoma), which have the potential for AFP production to be de-repressed, as well as in carcinoma of the lung ${ }^{3}$. Interestingly, patients with GCTs have been reported to have false elevations of AFP, secondary to liver damage caused by chemotherapy, anaesthetic agents or other drugs. ${ }^{28}$ Without awareness of such possibilities, patient management could potentially be altered based on such results. Thus, interpretation of AFP levels should only be undertaken with full knowledge of the patient's history and examination findings, including age, symptoms, past medical and family history and any 
relevant drug history ${ }^{22}$. In patients in whom potential liver disease is a concern, a full serum liver function test should be performed alongside AFP estimation ${ }^{22}$.

HCG measurements can be of the free $\beta$-subunit alone (monomer), the $\alpha-\beta$ dimer ('intact HCG'), or both ('total HCG', often referred to as ' $3-H C G$ ') ${ }^{3}$. HCG is measured with a double antibody immunometric assay ${ }^{3}$, typically using lanthanide fluorescence. Normal HCG levels are <2 IU/l, although levels can be measured in $\mathrm{ng} / \mathrm{ml}$ (where $5 \mathrm{IU} / \mathrm{l}=1 \mathrm{ng} / \mathrm{ml}$ ). The half-life of HCG is much shorter than AFP, usually $\sim 12-36 \mathrm{~h}^{3,29}$, and, therefore, levels should decay much more rapidly. HCG is produced primarily by CHC components, but low levels of HCG production can be observed in patients with pure seminoma containing syncytiotrophoblast (placental-like) cells (Table 1). However, even patients with advanced-stage pure seminoma only show raised HCG values in $<20 \%$ of cases ${ }^{3}$. HCG, like AFP, also shows limitations in sensitivity and specificity. Levels can also be elevated in other malignancies, including neuroendocrine, bladder, renal and lung carcinomas ${ }^{3}$. The treating clinician needs to be aware of the limited sensitivity of both HCG and AFP to ensure appropriate patient management.

LDH is an enzyme that catalyses the conversion of lactate to pyruvate ${ }^{3}$ and is ubiquitously expressed in cells of the body. Elevations in LDH are therefore very nonspecific, but can indicate increased cell turnover, such as in malignancy, including testicular GCTs. The half-life of LDH in the bloodstream is not well reported as serial measurements are not usually taken, but is measured in days rather than hours. An enzymatic assay measures circulating levels, but assays used between laboratories are 
very variable. LDH is often routinely measured at the time of cancer diagnosis, providing a surrogate measure for tumour bulk, and high levels are associated with an increased risk

of tumour lysis syndrome, which can occur in patients with malignant GCTs ${ }^{30}$. Increased levels are also observed in lymphoma, lung cancer and bone tumours such as osteosarcoma and Ewing sarcoma, as well as in any non-malignant condition that results in cellular lysis or injury, such as such as myocardial infarction or liver or muscle damage or disease ${ }^{3}$. These variations limit the use of LDH as a biomarker.

\section{[H2] Diagnosis and early follow-up monitoring}

[H3] Preorchidectomy. Measurement of CTM levels before orchidectomy is advised in all cases, as a substantially elevated preoperative AFP level precludes a diagnosis of pure seminoma, regardless of histological findings, and, in addition, this approach assists the interpretation of postorchidectomy estimations ${ }^{3}$. Furthermore, in rare cases, such as testicular GCTs with considerable tumour burden where urgent treatment is required, or extragonadal cases (such as retroperitoneal or mediastinal primary), in which the risks of surgical biopsy are deemed excessive, a substantially raised AFP and/or HCG might be sufficient for diagnosis ${ }^{3}$.

[H3] Stage I disease. Stage I testicular GCT is defined by the absence of demonstrable disease elsewhere on radiological imaging (that is, the disease is confined to the testis) and either normal levels of AFP and HCG, or if these levels were raised preoperatively, normalization following surgery. However, a proportion of patients with stage I 
malignant GCTs will develop clinical relapse during follow-up monitoring, and therefore for some patients, postorchidectomy risk stratification is undertaken.

Patients with NSGCTs are one such group whererisk stratification is usually undertaken; those at low risk of subsequent relapse are monitored with surveillance and patients with high-risk disease might be offered adjuvant therapy rather than surveillance. Effective adjuvant treatments that substantially reduce relapse risk in this setting include singledose carboplatin or radiotherapy for stage I seminoma and one course of bleomycin, etoposide and cisplatin (BEP) chemotherapy for stage I NSGCT ${ }^{4}$. The most important and consistently reported risk factors for recurrence in patients with stage I NSGCT has been the presence of lymphovascular invasion $(\mathrm{LVI})^{5-7,9,10}$. Patients without LVI have a predicted $15-20 \%$ risk of recurrence; in those with LVI, this risk is $40-50 \%$. The percentage embryonal carcinoma in the resection specimen (\%EC) ${ }^{6,7,10}$ and rete testis invasion (RTI) ${ }^{6}$ have also been reported to affect recurrence rates. In one large study of more than 1,000 patients, the overall relapse risk at 5 years after orchidectomy was $12 \%$ with none of these risk factors, compared with $50 \%$ with all three of these risk factors (31\% for the cohort as a whole) ${ }^{6}$. A further study demonstrated that immunohistochemical expression of CXCL12 [also known as stromal cell-derived factor 1 (SDF1)], in stage I NSGCTs was associated with reduced relapse risk ${ }^{31}$, a finding that has now been independently validated and shown to offer additional risk stratification benefit over LVI alone ${ }^{7}$. The authors propose a new prognostic index using CXCL12 expression in addition to LVI and \%EC, with three proposed risk groups (low, with a 
$\sim 10 \%$ relapse risk; moderate, with a $\sim 30-40 \%$ relapse risk; and high, with a $\sim 70 \%$ relapse risk) ${ }^{7}$, although this index remains to be prospectively validated in a trial setting.

Risk stratification of patients with seminoma has been more controversial than for those with NSGCTs. A review of 638 patients identified tumour size, RTI and LVI as being risk factors for relapse on univariate analysis with tumour size and RTI remaining as risk factors on multivariate analysis ${ }^{32}$. A risk of relapse of $12 \%, 15 \%$ and $31 \%$ was identified if zero, one or two risk factors were present, respectively ${ }^{32}$. This prognostic index has never been fully validated and indeed, in an analysis of data from 685 patients with stage I seminoma, only tumour size was a significant risk factor for relapse ${ }^{33}$. Risk of relapse increased from $9 \%$ for a $1 \mathrm{~cm}$ diameter tumour to $26 \%$ for an $8 \mathrm{~cm}$ diameter tumour ${ }^{33}$. Accurate prediction of relapse risk in this patient group remains elusive.

Novel circulating biomarkers could offer considerable additional value and refinement to such stratification systems in patients with stage I disease. For instance, a marker that shows increased sensitivity for residual microscopic disease might substantially reduce the need for adjuvant therapy.

[H3] Metastatic disease. Chemotherapy is routinely administered to patients with stage II-IV testicular GCTs according to IGCCC prognostic criteria ${ }^{11}$ (Table 2). Current standard-of-care treatment is three courses of BEP chemotherapy or four cycles of etoposide and cisplatin (EP) for good-risk disease (60\% of patients; $91 \%$ 5-year overall survival) and four BEP cycles for intermediate-risk disease (26\% of patients; $79 \%$ overall 
survival) and high-risk disease (14\% of patients; $48 \%$ overall survival) patients ${ }^{11}$. By IGCCC definition, patients with pure seminoma have normal levels of AFP. In the pooled multivariate analysis however, LDH levels of more than double the institutional upper limit of normal (ULN) in the metastatic seminoma cohort were predictive of shorter progression-free survival (PFS) and overall survival, but the IGCCC classification successfully stratified patients into intermediate-risk (10\% of patients) and good-risk groups (90\% of patients) solely by the presence or absence of nonpulmonary visceral metastases, respectively ${ }^{11}$. Thus, in patients with metastatic seminoma, the level of LDH and HCG does not affect their prognosis or treatment ${ }^{11}$. For those with NSGCT, however, AFP, HCG and LDH all have a role in determining IGCCC risk group (Table 2) ${ }^{11}$. The good-risk NSGCT group comprises patients with diagnostic serum levels of AFP $<1,000 \mathrm{ng} / \mathrm{ml}$, HCG $<5,000 \mathrm{IU} / \mathrm{l}$ and LDH $<1.5$ times the ULN ${ }^{11}$. The intermediategroup values are AFP $>1,000$ but $\leq 10,000 \mathrm{ng} / \mathrm{ml}$, HCG $>5,000$ but $\leq 50,000 \mathrm{IU} / \mathrm{l}$ or $\mathrm{LDH}$ $>1.5$ times but $\leq 10$ times the ULN. Finally, the poor-risk group comprises those patients with any CTM values above the upper limit of the intermediate-risk group range, plus patients with a mediastinal primary and/or nonpulmonary visceral metastases (Table 2) ${ }^{11}$. For patients who are AFP-positive and/or HCG-positive at diagnosis, measurement of AFP and/or HCG levels is recommended at the start of each chemotherapy cycle, in order to monitor the effectiveness of treatment. AFP and HCG should be measured at the end of treatment in all patients, as $\sim 50 \%$ of patients with metastatic seminoma who have an elevated HCG after chemotherapy experience subsequent relapse ${ }^{34,35}$ and some relapses manifest as NSGCTs ${ }^{3}$. End-of-treatment AFP and HCG levels generally do not assist in the identification of patients who could avoid retroperitoneal lymph node dissection or 
resection of residual tumour masses - surgery should go ahead unless CTMs are increasing, in which case a change to alternative chemotherapy is likely to be indicated ${ }^{3}$.

Measurement of serum AFP levels and/or decline might in the future help to further segregate IGCCC cohorts. One study of patients with intermediate-risk disease used AFP levels to further separate this population into two groups, with the small percentage of patients with values $>6,200 \mathrm{ng} / \mathrm{ml}$ having an overall survival similar to the IGCCC poorrisk group, whereas those with values less than this threshold had outcomes that approximated to those in the IGCCC good-risk group ${ }^{36}$. This suggestion remains to be validated prospectively. Clinical outcomes remain suboptimal for the $\sim 15 \%$ of patients with IGCCC poor-risk disease ${ }^{11}$. A previous attempt to further segregate the poor-risk group using the statistical technique of tree modelling was unsuccessful ${ }^{37}$. Furthermore, despite more than three decades of randomized controlled trials of treatment escalation (such as the addition of multi-agent chemotherapy ${ }^{38}$, taxols ${ }^{39}$, or high-dose therapy ${ }^{40}$, very few studies have demonstrated any clear additional survival benefit for such approaches. However, the early rate of decline of serum AFP and HCG levels has been shown to be of additional prognostic value in poor-risk patients ${ }^{41}$. Tumour markers were measured just before initiation of chemotherapy and again 3 weeks later (i.e. between days 18-21 inclusive), and rate of decline was expressed as predicted time to normalization (TTN) ${ }^{41}$. The 4-year PFS rates were $44 \%$ for the poor-risk group as a whole, but $64 \%$ and $38 \%$ in patients who had a favourable and an unfavourable TTN, respectively, independent of initial marker levels, disease site and the presence of nonpulmonary visceral metastases ${ }^{41}$. Early TTN was thus identified as an independent 
prognostic factor in poor-risk patients ${ }^{41}$. The prospective multicentre study GETUG13, which reported results in 2014, based treatment intensification on this early TTN and showed improved 3-year PFS in a randomized comparison of a dose-dense schedule versus standard BEP in patients with unfavourable marker decline (59\% versus $48 \%$, respectively) ${ }^{42}$. The 3 -year PFS in the small number of patients remaining in the favourable marker decline group was $70 \%{ }^{42}$. As a result, the GETUG13 study has been suggested to be a practice-changing step in the management of these NSGCTs ${ }^{43}, 44$. However, some concerns exist with this approach. Firstly, the timing of the reassessment of AFP level is strict and the calculation of the rate of decline in clinical practice can be challenging. For example, an AFP 'surge' or 'flare' on day 8 (compared with levels on day 1) following initiation of chemotherapy in patients with NSGCTs has been shown to be of adverse prognostic significance ${ }^{45}$, and such a phenomenon does not, therefore, simply represent a marker of tumour lysis ${ }^{45}$. As a result, interpretation of AFP levels at the beginning of the second course of chemotherapy in patients displaying an AFP flare should be undertaken with appropriate caution ${ }^{3}$. The effect of early AFP flares on the GETUG13 results is not clear. Secondly, the majority of patients (80\%) on the GETUG13 trial displayed unfavourable TTN of tumour markers ${ }^{41}$, and if these results of dose-dense schedules are adopted and replicated prospectively in other studies, most patients will receive treatment intensification with the potential for only modest benefit in PFS. Thirdly, even the identified 'good-risk' group within this poor-risk study had only a 70\% 3-year PFS, suggesting that, even in this cohort, outcomes are suboptimal. Finally, some patients in the poor-risk group were successfully treated by BEP alone, suggesting that improved prognostication would be valuable. 
AFP and/or HCG values that show an increase during therapy (aside from an initial surge or flare), usually indicate (YST-containing and/or CHC-containing) resistant or refractory disease and warrant a change in management ${ }^{3}$. Novel circulating biomarkers to better assist identification of all truly cisplatin-refractory cases upfront, either at the time of diagnosis or during early treatment (in both IGCCC intermediate-risk and poor-risk cohorts) would therefore be welcomed ${ }^{20}$.

\section{[H2] Detection of testicular GCT recurrence}

In patients with stage I testicular GCT disease, active surveillance after orchidectomy has been shown to lead to excellent outcomes (5-year disease-specific survival of $99.7 \%{ }^{9}$. Close monitoring of such patients not receiving adjuvant chemotherapy is needed, as a proportion harbour occult metastases, leading to subsequent relapse ${ }^{7}$. Approximately 20$30 \%$ of NSGCT and $10-20 \%$ of seminoma cases, respectively, relapse in this way ${ }^{6,7,9}$. The vast majority (92\%) of NSGCT relapses have occurred by 2 years of follow-up monitoring ${ }^{9}$. Such relapse patterns and frequency guide the intervals for CTM estimation during follow-up monitoring. The American Society of Clinical Oncology Clinical Practice Guideline recommends that follow-up assessment includes measurement of AFP and HCG levels in patients with stage I, chemotherapy-naive NSGCTs ${ }^{3}$. Markers are most commonly measured monthly during the first year of follow-up and every 2 months in the second year, with frequency reduced further thereafter ${ }^{3}$. Following a diagnosis of stage I pure seminoma, of those patients who will ultimately experience a relapse, 75\% will have done so by 2 years and $92 \%$ by 3 years $^{9}$. However, owing to the lack of 
sensitive markers, evidence for the clinical benefit of CTM estimation during follow-up visits is lacking in this population, and, therefore, not recommended ${ }^{3}$. For example, a 2015 study showed that only 3\% of stage I seminoma recurrences were detected by CTM evaluation, and even for stage I NSGCT cohorts, more than half of all relapses were negative by CTM estimation ${ }^{9}$. In the latter groups, CTM evaluation was only positive in $60 \%$ and $41 \%$ of stage I NSGCT patients with and without LVI, respectively ${ }^{9}$, explaining the reliance on 3D imaging in follow-up assessment and the highlighting the clinical need for markers of increased sensitivity and specificity for malignant GCT disease.

For patients with metastatic NSGCT after chemotherapy, follow-up measurement of both AFP and HCG is recommended, with the same interval and duration of follow-up as for stage I disease. For patients with advanced stage seminomas, $40 \%$ of whom have elevated HCG at diagnosis, follow-up measurement of CTMs is also recommended ${ }^{3}$. Such followup monitoring is recommended as rising CTM levels can represent the earliest sign of malignant GCT recurrence or relapse, before clinical or radiological evidence is present, ${ }^{46}$ although most clinicians would wait to see evidence of disease on imaging findings, before instigating appropriate management changes. Owing to the lack of direct evidence for their clinical benefit (for example, improved overall survival), recommended intervals for CTM estimation following treatment of advanced seminoma are generally less frequent than for NSGCT, typically every 2 months during the first year of follow-up monitoring, every 4 months during the second year, and less frequently thereafter ${ }^{3}$. In addition, no clear evidence indicates that LDH measurement is useful for the detection of recurrence during follow-up of patients with testicular GCT (including those with low- 
stage or advanced-stage, NSGCT or seminoma), owing to its lack of specificity ${ }^{47,48}$, and, therefore, such evaluation is not advised ${ }^{3}$.

\section{[H1] Other potential markers and investigations}

As AFP, HCG and LDH are not universal markers of malignant GCTs, researchers have attempted to identify other potential candidate blood-based biomarkers and investigations for GCTs.

\section{[H2] Circulating XIST transcripts}

Demethylated promoter regions of the long noncoding RNA X inactive specific transcript (XIST) gene at Xq13.2 have been reported in the plasma of men with testicular GCTs ${ }^{49}$. However, the 2004 study showing this finding was small, it only demonstrated an overall sensitivity of $64 \%$ for malignant testicular $\mathrm{GCTs}^{49}$ and the finding has not been confirmed by other reports. Thus, XIST promoter demethylation currently lacks the sensitivity and specificity required for a clinical test. Consequently, an urgent need remains to identify highly sensitive and specific serum markers for all malignant testicular GCTs, particularly for the detection of seminoma and embryonal carcinoma subtypes, which are predominantly marker-negative by CTM estimation.

\section{[H2] Circulating mRNA transcripts}

No reports have described circulating protein-coding mRNA transcripts as potential biomarkers in any malignant GCTs. Translation of any such candidate mRNA biomarker into the clinic would also be difficult, as mRNAs display relative instability at room temperature and in samples stored suboptimally ${ }^{50,51}$. 


\section{[H2] PET scans}

${ }^{18}$ F-fluorodeoxyglucose (FDG)-PET scans show better sensitivity and specificity for detecting active disease in seminoma masses after chemotherapy than does anatomical CT imaging, and consequently has become standard-of-care ${ }^{52,53} \cdot{ }^{18}$ FDG-PET might also have a role in detecting sites of relapse in patients with rising CTM levels after

chemotherapy ${ }^{54}$. However, despite encouraging pilot data, ${ }^{18}$ FDG-PET was insufficiently sensitive in patients with stage I high-risk LVI-positive NSGCT to identify a substantial proportion of patients who subsequently relapsed ${ }^{55}$. Likewise, this imaging modality has not been useful in predicting the histology of residual masses in patients with NSGCT treated with surgery. A full description of the role of PET scans in testicular GCT is outside the scope of this Review.

\section{[H1] MicroRNAs in diagnosis and monitoring}

The discovery of biological abnormalities that are common to all malignant GCTs is clinically relevant. Such abnormalities are likely to be critically important in disease pathogenesis, offer potential as biomarkers and might assist the identification of novel therapeutic targets in this disease ${ }^{20}$. MicroRNAs are short, non-protein-coding RNAs that regulate the expression of protein-coding genes. MicroRNAs are dysregulated in cancer, but expression profiles retain the characteristics of the cell of origin ${ }^{20}$. The first report of microRNA expression in malignant GCTs showed that the miR-371-373 microRNA cluster (at chromosomal location 19q13) was highly expressed in adult testicular disease, and might function as a potential oncogene through inhibition of LATS2 ${ }^{56}$. Subsequently, 
the specific overexpression of the miR-371-373 cluster in testicular GCTs was confirmed in a study that also included some adult ovarian malignant GCT cases $^{57}$. These findings were then extended in a report demonstrating that the miR-371-373 cluster and, in addition, the miR-302-367 cluster (miR-302a-d plus miR-367; at 4q25) were both overexpressed in all malignant GCTs, independent of patient age (paediatric or adult), tumour histological subtype (YST, seminoma or embryonal carcinoma) or anatomical site (gonadal or extragonadal) ${ }^{58}$, representing the first universal molecular abnormality identified in this disease ${ }^{20}$. Across $>100$ clinical cases, the expression levels of the eight main microRNA members from the miR-371-373 and miR-302-367 clusters accurately separated malignant GCTs from nonmalignant samples, comprising normal gonadal control samples and teratomas (Fig 1$)^{58}$ These findings suggest that microRNAs from the miR-371-373 and miR-302-367 clusters are potential highly sensitive and specific universal biomarkers of all malignant GCTs ${ }^{20}$. These observations have now been independently confirmed ${ }^{59}$, including the observation that these microRNA changes occur in germ cell neoplasia in $\operatorname{situ}^{60}$, the precursor lesion to testicular GCTs, implying that overexpression of these microRNAs represents an early molecular change that is likely to have a fundamental role in GCT pathogenesis. Importantly, these microRNAs have not been shown to be co-ordinately overexpressed in any other cancer or disease state, adding to their biomarker potential ${ }^{58}$.

MicroRNAs are released into the bloodstream from cancer cells, often contained within membrane-bound particles, termed exosomes, and are therefore protected from degradation $^{61,62}$. Consequently, the quantification of circulating microRNAs offers 
substantial promise for cancer diagnosis and monitoring ${ }^{63}$. For malignant GCTs, a multiplexed quantitative reverse transcription (qRT)-PCR methodology approach was used to demonstrate that circulating levels of all eight main members of the miR-371-373 and miR-302-367 clusters were elevated in the serum of an index patient with extragonadal disease, compared with levels in pooled normal serum ${ }^{63}$. Levels of miR-372-3p fell to normal levels during treatment and during uneventful clinical follow-up monitoring ${ }^{63}$. This proof-of-principle was followed by a small study across the clinical spectra of age (paediatric and adult), anatomical site [gonadal (including testicular) and extragonadal] and histological subtype (YST, seminoma and embryonal carcinoma), confirming universal elevation at malignant GCT diagnosis of serum levels of miR-372-3p and miR-367-3p ${ }^{64}$. Importantly, most of the patients described in this study were marker-negative by CTM estimation (specifically the seminoma and embryonal carcinoma cases), demonstrating potential clinical utility in these cohorts.

These initial findings have since been replicated and extended, based on the qRT-PCR methodology described ${ }^{12,13,64-70}$, predominantly in malignant testicular GCTs. Indeed, a panel of just four circulating microRNAs (namely miR-371a-3p, miR-372-3p, miR-373-3p and miR367-3p) is highly sensitive and specific for diagnosis of malignant GCT ${ }^{12,66,70}$. Circulating microRNA levels from the panel fall after definitive surgery and/or chemotherapy treatment ${ }^{12}$, 13, 63, 66, 70 , and are also highly sensitive for detecting relapse ${ }^{12}$.

\section{[H2] Practical considerations for microRNA testing}


Circulating microRNAs are now starting to be be studied in prospective clinical trials in patients with GCTs, with the ultimate aim of embedding microRNA quantification in routine clinical practice. However, at present no agreed protocol exists for sample collection, RNA extraction, quality control assessment or actual PCR quantification, and indeed, the full panel of four microRNAs that might offer the greatest sensitivity and specificity for detecting all malignant testicular GCTs has not always been quantified (Table 3). These issues are all important and need to be overcome to ensure optimal reliability and reproducibility and to drive these research technologies towards future clinical use in testicular GCTs.

\section{[H3] Sample collection.}

To date, all studies specifically reporting circulating microRNA levels from the miR371-373 and miR-302-367 clusters in malignant GCTs have used serum ${ }^{12,13,63-70}$. To ensure consistent results, the whole blood collected in these serum separator tubes should be centrifuged in a standard fashion within a few hours following receipt, and the resultant serum aliquoted into separate tubes for processing, with subsequent storage at $80^{\circ} \mathrm{C}$. The starting serum volumes used for testing have reduced in recent years. Initially, $400 \mu 1$ was used $^{63,64} ; 200 \mu 1$ is now the most common starting volume, but as little as $50 \mu \mathrm{l}$ can be sufficient ${ }^{66,67}$ (Table 3). Plasma might also be suitable for testing, as serum and plasma microRNA levels are believed to correlate well ${ }^{71}$, although such a correlation remains to be formally demonstrated for the panel of four circulating microRNAs at the time of malignant GCT diagnosis. If proven to be suitable, the use of plasma would offer the additional benefit of enabling any circulating tumour DNA (ctDNA) that is present to 
be extracted, which is important as mutational profiles in testicular GCTs using wholeexome sequencing have now been reported ${ }^{72}$. Mutations occurred in $43 \%$ of the tumours interrogated, with aberrations in the KIT gene (occurring in $14.3 \%$ of tumours) being the most common. Of particular interest, two treatment-refractory cases were identified, both of which harboured XRCC2 mutations, a gene implicated in cisplatin resistance ${ }^{72}$. Collection of plasma in prospective studies might, therefore, offer further opportunities for the molecular diagnosis and risk stratification of malignant GCTs. [H3] RNA extraction.

RNA is extracted from serum using commercially available proprietary kits (Table 3). The subsequent PCR reactions are also performed using standard TaqMan assay kits and platforms (Table 3). To avoid false-positive and false-negative results, no-template controls should be run for each assay (to exclude nonspecific amplification) and any differences in RNA extraction efficiency between samples measured, so that these differences can be accounted for in subsequent data analysis ${ }^{73}$. The latter is performed by adding a fixed quantity of exogenous nonhuman spike-in microRNA(s) (for example, $C$. elegans (cel)-miR-39-3p or A. thaliana (ath)-miR-159-3p), that does not occur naturally in human serum, which is then quantified and adjusted for. Not all reported studies use such a quality control step (Table 3), despite it providing additional stringency and being considered best practice $^{12}$, as per Minimal Information in Q-PCR Experiment (MIQE) guidelines $^{73}$. MIQE guidelines assist both the transparency of result reporting and the comparison of results between different studies, which may facilitate the subsequent standardization of protocols ${ }^{73}$. The selection of endogenous circulating microRNA housekeeping genes for data normalization (relative quantification) is also of critical 
importance, as this step minimizes technical bias and maximizes detection of true biological variation between serum samples ${ }^{12}$. Early studies did not include such normalization steps ${ }^{63,64}$, as no stable housekeeping microRNAs had been established. In many subsequent reports, no clear rationale is given for the selection of different housekeeping genes for this purpose (Table 3). However, one MIQE-compliant 2016 report has identified miR-30b-5p as being the most stable microRNA in serum, based on a global microRNA study $(n=741)$ and subsequent validation in serum from patients with malignant GCTs ${ }^{12}$. Some researchers suggest that such relative quantification might be unnecessary for clinical testing and that raw PCR results provide adequate diagnostic discrimination ${ }^{69}$. However, such an approach may not be optimal when using current qRT-PCR methodology to quantify the full panel of four circulating microRNAs that offer the greatest accuracy for malignant GCT diagnosis ${ }^{12}$. The dynamic range for circulating miR-372-3p, miR-373-3p and miR-367-3p is lower than for circulating miR$371 a-3 p^{12}$. Consequently, differences in extraction efficiency will have a relatively high impact on overall PCR measurements and, therefore, careful control via relative quantification approaches is still recommended ${ }^{12}$.

[H3] Haemolysis.

Haemolysis might also be an important practical consideration in adopting serum microRNA tests for routine clinical detection of testicular malignant GCTs, as variations in red blood cell lysis between serum and plasma samples might result in altered circulating microRNA expression levels for technical, rather than biological, reasons. Consequently, a 2016 study only used samples without macroscopic evidence of haemolysis, and calculated $\Delta \mathrm{Ct}$ (miR-23a-3p minus miR-451a) values to assess 
differences in microscopic haemolysis between samples ${ }^{12}$ (Table 3). MiR-23a-3p quantification is used as a housekeeping gene to enable a $\Delta \mathrm{Ct}$ value to be estimated, and miR-451a levels directly reflect the degree of haemolysis of the sample being interrogated, as this microRNA is one of the most abundant in red blood cells ${ }^{12}$. Other methods, such as spectrophotometry for quantification of free haemoglobin in the serum, can quantify the degree of haemolysis within a sample. The value of such assessments in circulating microRNA testing remains to be established for the diagnosis of malignant GCT. However, we recommend that a formal method of haemolysis assessment should continue to be performed at present, to inform how much contribution haemolysis makes to any potential false positive results in future trials of circulating microRNAs in malignant GCTs, and to enable appropriate adjustments to be made.

[H3] Using the full panel of four microRNAs.

For maximal sensitivity and specificity, using the full panel of four microRNAs from the miR-371-373 and miR-302-367 clusters is advisable ${ }^{12,66,70}$. The sensitivity of miR371a-3p alone is generally $~ 90 \%$ for the diagnosis of malignant $\mathrm{GCT}^{68}$, but sensitivity and specificity can be increased further by using the panel ${ }^{12,} 66,70$. For example, the particularly rapid reduction in miR-371a-3p levels following orchidectomy in stage I disease $^{68}$ can result in negative results in samples taken more than one day after surgery, whereas the other microRNAs in the panel have longer half-lives ${ }^{12}$. Accordingly, in cases in which the timing of the 'diagnostic' serum sample is suboptimal (for example, taken following surgery), assessment of miR-371a-3p alone might result in (false) negative results. Furthermore, assessment of circulating levels of miR-367-3p increases specificity for the identification of malignant GCTs, as it is transcribed from a separate chromosome 
locus (4q25) to the other three microRNAs in the panel (19q13) ${ }^{12}$. Such assessment is important, as although individual microRNAs from either the miR-371-373 or the miR302-367 cluster have been shown to be increased in certain malignancies, coordinate overexpression of microRNAs from both clusters have not been demonstrated in any tumour other than malignant GCTs, or in any other disease state ${ }^{57}$. We have proposed a pipeline for the quantification of circulating microRNAs in malignant GCTs that addresses these issues (Fig 2).

Very recently, two further serum microRNA studies in testicular GCTs have been published (refs 73 and 74 - van Agthoven and Looijenga PMID 27487133 and Dieckmann et al PMID 27495845). Both showed that the panel of miR-371-373 and miR-367-3p microRNAs was significantly elevated in patients with malignant testicular GCTs at the time of diagnosis, using the originally described pre-amplification step (ref 63), which maximises sensitivity of the test. MiR-371a-3p was the most sensitive and specific individual microRNA from the serum panel in these analyses, with area under the curve (AUC) values of 0.95 and 0.94, respectively (refs 73 and 74 - van Agthoven and Looijenga PMID 27487133 and Dieckmann et al PMID 27495845). One study did not use an exogenous nonhuman spike-in microRNA and used miR-93-5p for normalisation (ref 74 Dieckmann et al). The other used a nonhuman spike-in and selected miR-30b-5p as the housekeeping gene (ref 73 van Agthoven and Looijenga), as previously described (ref 12). Interestingly, this was because miR-93-5p was found to be unsuitable for normalisation due to a significant difference in levels between the 
malignant GCT group and the control patients (ref 73 van Agthoven and Looijenga). Neither study used a formal haemolysis method to assess the samples (Table 3). [H3] Cost and the future.

Currently, as a relatively low-throughput research method using the described pipeline (Fig 2$)^{12}$, circulating microRNA analysis costs approximately UK£40 per sample. This figure compares favourably with the cost of a single CT scan of $\sim £ 200$. In due course, when such a method is in routine clinical use with higher throughput, economies of scale are likely to reduce these costs further. In the future, robust absolute quantification PCR methods might overcome the need for relative quantification when measuring circulating microRNA levels, although the requirement to use nonhuman spike-ins or other appropriate standards will remain, to ensure the accuracy of RNA extraction steps and subsequent PCR quantification within and between samples. Such measures should improve sensitivity and specificity overall and provide greater confidence in borderline cases, ensuring that clinical decisions are not undermined by subtle technical effects ${ }^{12}$.

\section{[H1] Conclusions}

The conventional tumour markers AFP, HCG and LDH have demonstrated value in the clinical management of testicular malignant GCTs, particularly for early riskstratification of metastatic NSGCTs and detection of relapse in patients with NSGCT. However, their limitations in sensitivity and specificity prevent more universal application, especially in patients with seminoma. Circulating microRNAs show exciting promise as a universal marker of testicular malignant GCTs. Such biomarkers could considerably contribute to improving the quality of survival for patients in low-stage and/or IGCCC good-risk groups, through rational reductions in adjuvant chemotherapy, 
improved targeted imaging and detection of relapse at an earlier stage. Their use might also enable new approaches to increase overall survival among IGCCC poor-risk and relapsed disease cohorts by modifying treatment and identifying patients who should receive intensified treatment.

As these novel biomarkers are now being studied in prospective clinical trials, the practical considerations discussed in this Review should be addressed and incorporated into standardized protocols. We suggest that haemolysis assessment, alongside normalization to both a nonhuman exogenous spike-in RNA (e.g. cel-miR-39-3p) and carefully selected endogenous housekeeping microRNAs (e.g. miR-30b-5p), are optimal approaches for the relative quantification PCR methods currently in use. Such robust protocols will ensure the optimal reliability and reproducibility of these tests in clinical settings. 


\section{Acknowledgements}

The authors would like to acknowledge grant funding from CwCUK/GOSHCC (M.J.M. N.C. grant W1058), SPARKS (M.J.M. N.C. grant 11CAM01), CRUK (N.C. grant A13080) MRC (M.J.M. grant MC_EX_G0800464) and National Health Service funding to the Royal Marsden/Institute of Cancer Research National Institute for Health Research Biomedical Research Centre for Cancer (R.A.H.). The authors also thank the Max Williamson Fund, the Josh Carrick Foundation and The Perse Preparatory School, Cambridge for support.

\section{Competing interests}

M.J.M. and N.C. receive laboratory funding from AstraZeneca as part of a collaborative research programme investigating clinical applications of circulating microRNA quantification. R.A.H. declares no competing interests. 


\section{References}

1. Trabert, B., Chen, J., Devesa, S.S., Bray, F. \& McGlynn, K.A. International patterns and trends in testicular cancer incidence, overall and by histologic subtype, 1973-2007. Andrology 3, 4-12 (2015).

2. Moch, H., Humphrey, P.A., Ulbright, T.M. \& Reuter, V.E. in IARC WHO Classification of Tumours, Fourth Edition (ed. World Health Organization) (2016).

3. Gilligan, T.D. et al. American Society of Clinical Oncology Clinical Practice Guideline on uses of serum tumor markers in adult males with germ cell tumors. $J$ Clin Oncol 28, 3388-404 (2010).

4. de Wit, R. \& Fizazi, K. Controversies in the management of clinical stage I testis cancer. J Clin Oncol 24, 5482-92 (2006).

5. Cohn-Cedermark, G., Stahl, O., Tandstad, T. \& Swenoteca. Surveillance vs. adjuvant therapy of clinical stage I testicular tumors - a review and the SWENOTECA experience. Andrology 3, 102-10 (2015).

6. Daugaard, G. et al. Surveillance for stage I nonseminoma testicular cancer: outcomes and long-term follow-up in a population-based cohort. J Clin Oncol 32, 3817-23 (2014).

7. Gilbert, D.C. et al. Defining a New Prognostic index for Stage I Nonseminomatous Germ Cell Tumors using CXCL12 Expression and Proportion of Embyronal Carcinoma. Clin Cancer Res (2015). 
8. Kobayashi, K. et al. Oncological outcomes in patients with stage I testicular seminoma and nonseminoma: pathological risk factors for relapse and feasibility of surveillance after orchiectomy. Diagn Pathol 8, 57 (2013).

9. Kollmannsberger, C. et al. Patterns of relapse in patients with clinical stage I testicular cancer managed with active surveillance. J Clin Oncol 33, 51-7 (2015).

10. Lago-Hernandez, C.A. et al. A refined risk stratification scheme for clinical stage 1 NSGCT based on evaluation of both embryonal predominance and lymphovascular invasion. Ann Oncol 26, 1396-401 (2015).

11. International Germ Cell Consensus Classification: a prognostic factor-based staging system for metastatic germ cell cancers. International Germ Cell Cancer Collaborative Group. J Clin Oncol 15, 594-603 (1997).

12. Murray, M.J. et al. A pipeline to quantify serum and cerebrospinal fluid microRNAs for diagnosis and detection of relapse in paediatric malignant germcell tumours. Br J Cancer 114, 151-62 (2016).

13. Dieckmann, K.P. et al. MicroRNAs miR-371-3 in serum as diagnostic tools in the management of testicular germ cell tumours. Br J Cancer 107, 1754-60 (2012).

14. Tarin, T.V., Sonn, G. \& Shinghal, R. Estimating the risk of cancer associated with imaging related radiation during surveillance for stage I testicular cancer using computerized tomography. J Urol 181, 627-32; discussion 632-3 (2009).

15. Fung, C., Fossa, S.D., Milano, M.T., Oldenburg, J. \& Travis, L.B. Solid tumors after chemotherapy or surgery for testicular nonseminoma: a population-based study. J Clin Oncol 31, 3807-14 (2013). 
16. Haugnes, H.S. et al. Long-term and late effects of germ cell testicular cancer treatment and implications for follow-up. J Clin Oncol 30, 3752-63 (2012).

17. Haugnes, H.S. et al. Cardiovascular risk factors and morbidity in long-term survivors of testicular cancer: a 20-year follow-up study. J Clin Oncol 28, 464957 (2010).

18. Huddart, R.A. et al. Cardiovascular disease as a long-term complication of treatment for testicular cancer. J Clin Oncol 21, 1513-23 (2003).

19. Kollmannsberger, C. et al. Late toxicity following curative treatment of testicular cancer. Semin Surg Oncol 17, 275-81 (1999).

20. Murray, M.J., Nicholson, J.C. \& Coleman, N. Biology of childhood germ cell tumours, focussing on the significance of microRNAs. Andrology 3, 129-139 (2015).

21. Travis, L.B. et al. Second cancers among 40,576 testicular cancer patients: focus on long-term survivors. J Natl Cancer Inst 97, 1354-65 (2005).

22. Murray, M.J. \& Nicholson, J.C. alpha-Fetoprotein. Arch Dis Child Educ Pract Ed 96, 141-7 (2011).

23. Gitlin, D., Perricelli, A. \& Gitlin, G.M. Synthesis of -fetoprotein by liver, yolk sac, and gastrointestinal tract of the human conceptus. Cancer Res 32, 979-82 (1972).

24. Nishizono, I. et al. Rapid and sensitive chemiluminescent enzyme immunoassay for measuring tumor markers. Clin Chem 37, 1639-44 (1991). 
25. Blohm, M.E., Vesterling-Horner, D., Calaminus, G. \& Gobel, U. Alpha 1fetoprotein (AFP) reference values in infants up to 2 years of age. Pediatr Hematol Oncol 15, 135-42 (1998).

26. Johnson, P.J. Role of alpha-fetoprotein in the diagnosis and management of hepatocellular carcinoma. J Gastroenterol Hepatol 14 Suppl, S32-6 (1999).

27. Schneider, D.T., Calaminus, G. \& Gobel, U. Diagnostic value of alpha 1fetoprotein and beta-human chorionic gonadotropin in infancy and childhood. Pediatr Hematol Oncol 18, 11-26 (2001).

28. Germa, J.R., Llanos, M., Tabernero, J.M. \& Mora, J. False elevations of alphafetoprotein associated with liver dysfunction in germ cell tumors. Cancer 72, 2491-4 (1993).

29. Murray, M.J., Nicholson J.C. Germ Cell Tumours in Children and Adolescents. Paediatrics and Child Health 20, 109-116 (2010).

30. Murray, M.J. et al. Intra-abdominal metastasis of an intracranial germinoma via ventriculo-peritoneal shunt in a 13-year-old female. Br J Neurosurg 25, 747-9 (2011).

31. Gilbert, D.C. et al. Clinical and biological significance of CXCL12 and CXCR4 expression in adult testes and germ cell tumours of adults and adolescents. $J$ Pathol 217, 94-102 (2009).

32. Warde, P. et al. Prognostic factors for relapse in stage I seminoma managed by surveillance: a pooled analysis. J Clin Oncol 20, 4448-52 (2002).

33. Chung, P. et al. Evaluation of a prognostic model for risk of relapse in stage I seminoma surveillance. Cancer Med 4, 155-60 (2015). 
34. Miller, K.D., Loehrer, P.J., Gonin, R. \& Einhorn, L.H. Salvage chemotherapy with vinblastine, ifosfamide, and cisplatin in recurrent seminoma. J Clin Oncol 15, 1427-31 (1997).

35. Vuky, J. et al. Salvage chemotherapy for patients with advanced pure seminoma. J Clin Oncol 20, 297-301 (2002).

36. Raggi, D. et al. Prognostic reclassification of patients with intermediate-risk metastatic germ cell tumors: Implications for clinical practice, trial design, and molecular interrogation. Urol Oncol 33, 332 e19-24 (2015).

37. van Dijk, M.R., Steyerberg, E.W., Stenning, S.P. \& Habbema, J.D. Identifying subgroups among poor prognosis patients with nonseminomatous germ cell cancer by tree modelling: a validation study. Ann Oncol 15, 1400-5 (2004).

38. Huddart, R.A. et al. A randomised phase 2 trial of intensive induction chemotherapy (CBOP/BEP) and standard BEP in poor-prognosis germ cell tumours (MRC TE23, CRUK 05/014, ISRCTN 53643604). Eur Urol 67, 534-43 (2015).

39. de Wit, R. et al. Randomized phase III study comparing paclitaxel-bleomycin, etoposide, and cisplatin (BEP) to standard BEP in intermediate-prognosis germcell cancer: intergroup study EORTC 30983. J Clin Oncol 30, 792-9 (2012).

40. Daugaard, G. et al. A randomized phase III study comparing standard dose BEP with sequential high-dose cisplatin, etoposide, and ifosfamide (VIP) plus stemcell support in males with poor-prognosis germ-cell cancer. An intergroup study of EORTC, GTCSG, and Grupo Germinal (EORTC 30974). Ann Oncol 22, 105461 (2011). 
41. Fizazi, K. et al. Early predicted time to normalization of tumor markers predicts outcome in poor-prognosis nonseminomatous germ cell tumors. J Clin Oncol 22, 3868-76 (2004).

42. Fizazi, K. et al. Personalised chemotherapy based on tumour marker decline in poor prognosis germ-cell tumours (GETUG 13): a phase 3, multicentre, randomised trial. Lancet Oncol 15, 1442-50 (2014).

43. Killock, D. Chemotherapy. After 25 years, therapy for poor-prognosis GCTs advances. Nat Rev Clin Oncol 12, 3 (2015).

44. Lorch, A. A practice-changing step forward in germ-cell cancer? Lancet Oncol 15, 1409-10 (2014).

45. de Wit, R. et al. Serum alpha-fetoprotein surge after the initiation of chemotherapy for non-seminomatous testicular cancer has an adverse prognostic significance. Br J Cancer 78, 1350-5 (1998).

46. Grigor, K.M., Detre, S.I., Kohn, J. \& Neville, A.M. Serum alpha1-foetoprotein levels in 153 male patients with germ cell tumours. Br J Cancer 35, 52-8 (1977).

47. Ackers, C. \& Rustin, G.J. Lactate dehydrogenase is not a useful marker for relapse in patients on surveillance for stage I germ cell tumours. Br J Cancer 94, 1231-2 (2006).

48. Venkitaraman, R. et al. The utility of lactate dehydrogenase in the follow-up of testicular germ cell tumours. BJU Int 100, 30-2 (2007).

49. Kawakami, T., Okamoto, K., Ogawa, O. \& Okada, Y. XIST unmethylated DNA fragments in male-derived plasma as a tumour marker for testicular cancer. Lancet 363, 40-2 (2004). 
50. Rainen, L. et al. Stabilization of mRNA expression in whole blood samples. Clin Chem 48, 1883-90 (2002).

51. Viprey, V.F. et al. Standardisation of operating procedures for the detection of minimal disease by QRT-PCR in children with neuroblastoma: quality assurance on behalf of SIOPEN-R-NET. Eur J Cancer 43, 341-50 (2007).

52. Bachner, M. et al. 2-(1)(8)fluoro-deoxy-D-glucose positron emission tomography (FDG-PET) for postchemotherapy seminoma residual lesions: a retrospective validation of the SEMPET trial. Ann Oncol 23, 59-64 (2012).

53. De Santis, M. et al. 2-18fluoro-deoxy-D-glucose positron emission tomography is a reliable predictor for viable tumor in postchemotherapy seminoma: an update of the prospective multicentric SEMPET trial. J Clin Oncol 22, 1034-9 (2004).

54. Hain, S.F. et al. Fluorodeoxyglucose positron emission tomography in the evaluation of germ cell tumours at relapse. Br J Cancer 83, 863-9 (2000).

55. Huddart, R.A. et al. 18fluorodeoxyglucose positron emission tomography in the prediction of relapse in patients with high-risk, clinical stage I nonseminomatous germ cell tumors: preliminary report of MRC Trial TE22--the NCRI Testis Tumour Clinical Study Group. J Clin Oncol 25, 3090-5 (2007).

56. Voorhoeve, P.M. et al. A Genetic Screen Implicates miRNA-372 and miRNA-373 As Oncogenes in Testicular Germ Cell Tumors. Cell 124, 1169-1181 (2006).

57. Gillis, A.J.M. et al. High-throughput microRNAome analysis in human germ cell tumours. J Pathol 213, 319-328 (2007). 
58. Palmer, R.D. et al. Malignant germ cell tumors display common microRNA profiles resulting in global changes in expression of messenger RNA targets. Cancer Research 70, 2911-23 (2010).

59. Bing, Z. et al. MicroRNA expression profiles of seminoma from paraffinembedded formalin-fixed tissue. Virchows Arch 461, 663-8 (2012).

60. Novotny, G.W. et al. MicroRNA expression profiling of carcinoma in situ cells of the testis. Endocr Relat Cancer 19, $365-79$ (2012).

61. Caby, M.P., Lankar, D., Vincendeau-Scherrer, C., Raposo, G. \& Bonnerot, C. Exosomal-like vesicles are present in human blood plasma. Int Immunol 17, 87987 (2005).

62. Valadi, H. et al. Exosome-mediated transfer of mRNAs and microRNAs is a novel mechanism of genetic exchange between cells. Nat Cell Biol 9, 654-9 (2007).

63. Murray, M.J. et al. Identification of MicroRNAs From the miR-371 373 and miR-302 Clusters as Potential Serum Biomarkers of Malignant Germ Cell Tumors. Am J Clin Pathol 135, 119-25 (2011).

64. Murray, M.J. \& Coleman, N. Testicular cancer: a new generation of biomarkers for malignant germ cell tumours. Nat Rev Urol 9, 298-300 (2012).

65. Belge, G., Dieckmann, K.P., Spiekermann, M., Balks, T. \& Bullerdiek, J. Serum levels of microRNAs miR-371-3: a novel class of serum biomarkers for testicular germ cell tumors? Eur Urol 61, 1068-9 (2012). 
66. Gillis, A.J. et al. Targeted serum miRNA (TSmiR) test for diagnosis and followup of (testicular) germ cell cancer patients: A proof of principle. Mol Oncol 7, 1083-92 (2013).

67. Rijlaarsdam, M.A. et al. Identification of known and novel germ cell cancerspecific (embryonic) miRs in serum by high-throughput profiling. Andrology 3, 85-91 (2015).

68. Spiekermann, M. et al. MicroRNA miR-371a-3p in serum of patients with germ cell tumours: evaluations for establishing a serum biomarker. Andrology 3, 78-84 (2015).

69. Spiekermann, M., Dieckmann, K.P., Balks, T., Bullerdiek, J. \& Belge, G. Is Relative Quantification Dispensable for the Measurement of MicroRNAs as Serum Biomarkers in Germ Cell Tumors? Anticancer Res 35, 117-21 (2015).

70. Syring, I. et al. Circulating Serum miRNA (miR-367-3p, miR-371a-3p, miR-3723p and miR-373-3p) as Biomarkers in Patients with Testicular Germ Cell Cancer. J Urol 193, 331-7 (2015).

71. Exiqon. Biofluids guidelines: analyzing microRNAs in liquid biopsies. http://www.exiqon.com/ls/Documents/Scientific/microRNA-serum-plasmaguidelines.pdf. (2015). Accessed 2nd March 2016..

72. Litchfield, K. et al. Whole-exome sequencing reveals the mutational spectrum of testicular germ cell tumours. Nat Commun 6, 5973 (2015).

73. Bustin, S.A. et al. The MIQE guidelines: minimum information for publication of quantitative real-time PCR experiments. Clin Chem 55, 611-22 (2009). 
Figure 1 | Differential expression of the microRNAs (miR)-371-373 and miR-302367 clusters in malignant germ cell tumours (GCT). Hierarchical clustering analysis based on the eight main microRNAs from the miR-371-373 and miR-302-367 clusters (rows) segregates a | paediatric and $\mathrm{b}$ | adult malignant GCT samples from nonmalignant controls (comprising benign teratomas and normal gonadal controls) (columns). In the heatmap, red represents relative microRNA overexpression and blue represents underexpression. Green columns = normal gonadal controls; brown columns = teratoma; blue columns = seminoma; yellow columns = yolk sac tumour; red columns $=$ embryonal carcinoma.

Figure 2 | A proposed pipeline for quantification of circulating microRNAs (miRs) in malignant germ cell tumours (GCTs) ${ }^{12}$. At the RNA extraction stage (green box), a fixed quantity of the non-human spike-in RNA cel-miR-39-3p is added to enable subsequent exogenous normalisation. An initial quality control quantitative reverse transcription (qRT)-PCR step is then performed (blue box) to check for satisfactory levels of exogenous cel-miR-39-3p (indicating satisfactory RNA extraction), endogenous housekeeping microRNA (miR-30b-5p) and for haemolysis assessment. Once a serum sample has passed this step, formal microRNA quantification is then performed, using a multiplexed reverse transcription and preamplification step (purple box), which includes the panel of four test microRNAs (miR-371a-3p, miR-372-3p, miR-373-3p and miR-3673p). Data analysis is then undertaken (red box). 
Table 1 | Serum AFP and HCG levels generally observed in germ cell tumour subtypes (adapted from reference ${ }^{22}$ )

\begin{tabular}{|l|c|c|}
\hline GCT histological subtype & AFP & HCG \\
\hline Yolk sac tumour & ++ & - \\
\hline Seminoma & - & \pm \\
\hline Embryonal carcinoma & \pm & \pm \\
\hline Choriocarcinoma & - & ++ \\
\hline Teratoma & \pm & - \\
\hline
\end{tabular}

$++=$ strongly positive levels; $\pm=$ levels may be negative or moderately positive; = negative levels.

AFP, $\alpha$-fetoprotein; GCT, germ cell tumour; HCG, human chorionic gonadotrophin. 
Table 2 | Summary of AFP, HCG and LDH marker levels in the prognostic groups of the IGCCC classification (adapted from reference ${ }^{11}$ )

\begin{tabular}{|c|c|c|}
\hline Clinical variable & Seminoma & NSGCT \\
\hline \multicolumn{3}{|l|}{ Good-prognosis group } \\
\hline Primary site & Any & Testis or retroperitoneal \\
\hline Metastases & No NPVM & No NPVM \\
\hline AFP (ng/ml) & Normal & $<1,000$ \\
\hline HCG (IU/L) & Any & $<5,000$ \\
\hline LDH (xULN) & Any & $<1.5 \times$ xULN \\
\hline \multicolumn{3}{|c|}{$\begin{array}{c}\text { Intermediate-prognosis } \\
\text { group }\end{array}$} \\
\hline Primary site & Any & Testis or retroperitoneal \\
\hline Metastases & NPVM & No NPVM \\
\hline AFP (ng/ml) & Normal & $\geq 1,000$ and $\leq 10,000^{*}$ \\
\hline HCG (IU/L) & Any & $\geq 5,000$ and $\leq 50,000^{*}$ \\
\hline LDH $(x U L N)$ & Any & $\geq 1.5$ and $\leq 10 \times$ xULN $^{*}$ \\
\hline \multicolumn{3}{|l|}{ Poor-prognosis group } \\
\hline Primary site & \multirow{5}{*}{$\begin{array}{l}\text { No patients classified as } \\
\text { poor prognosis }\end{array}$} & $\begin{array}{l}\text { Mediastinal**; or testis or } \\
\text { retroperitoneal with any of } \\
\text { the risk factors below }\end{array}$ \\
\hline Metastases & & $\mathrm{NPVM}^{* *}$ \\
\hline AFP (ng/ml) & & $>10,000 * *$ \\
\hline HCG (IU/L) & & $>50,000^{* *}$ \\
\hline LDH (xULN) & & $>10 \times \mathrm{xUN}^{* *}$ \\
\hline
\end{tabular}

* = any one of these risk-factors will classify a NSGCT patient as intermediateprognosis;

** = any one of these risk-factors will classify a NSGCT patient as poor-prognosis.

AFP, $\alpha$-fetoprotein; GCT, germ cell tumour; HCG, human chorionic gonadotrophin. LDH, lactate dehydrogenase; NSGCT, non-seminomatous GCT; NPVM, non-pulmonary visceral metastases; ULN, upper limit of normal. 
Table 3 | Serum microRNA studies in malignant germ cell tumours (GCTs) and important practical considerations.

\begin{tabular}{|c|c|c|c|c|c|c|c|}
\hline $\begin{array}{l}\text { Author } \\
\text { (year) }\end{array}$ & $\begin{array}{l}\text { Serum } \\
\text { volume } \\
\text { used } \\
(\mu \mathrm{l})\end{array}$ & $\begin{array}{l}\text { RNA } \\
\text { extraction } \\
\text { method }\end{array}$ & $\begin{array}{c}\text { Test } \\
\text { microRNAs } \\
\text { or } \\
\text { microRNA } \\
\text { clusters } \\
\text { quantified }\end{array}$ & $\begin{array}{l}\text { Haemolysis } \\
\text { Assessment }\end{array}$ & $\begin{array}{l}\text { Non- } \\
\text { human } \\
\text { exogenous } \\
\text { spike-in } \\
\text { RNA } \\
\text { added }\end{array}$ & $\begin{array}{l}\text { Normalisation } \\
\text { approach }\end{array}$ & Comments \\
\hline $\begin{array}{l}\text { Murray et a. } \\
(2011) l^{63}\end{array}$ & 400 & $\begin{array}{l}\text { miRVana } \\
\text { PARIS kit } \\
\text { (Ambion) }\end{array}$ & $\begin{array}{c}\text { miR-371- } \\
373(\mathrm{n}=3) \\
\operatorname{miR}-302- \\
367(\mathrm{n}=5)\end{array}$ & No & No & $\begin{array}{c}\text { Small RNA } \\
\text { concentration }\end{array}$ & $\begin{array}{c}\text { First report of } \\
\text { utility of serum } \\
\text { microRNAs in } \\
\text { GCTs } \\
\end{array}$ \\
\hline $\begin{array}{l}\text { Belge et al. } \\
\text { (2012) }\end{array}$ & $\begin{array}{c}\text { Not } \\
\text { described }\end{array}$ & Not described & $\begin{array}{c}\mathrm{miR}-371- \\
373(\mathrm{n}=3)\end{array}$ & No & No & 18S RNA & $\begin{array}{l}\text { Letter, with } \\
\text { sparse technical } \\
\text { details }\end{array}$ \\
\hline $\begin{array}{l}\text { Murray and } \\
\text { Coleman } \\
(2012)^{64}\end{array}$ & 400 & $\begin{array}{l}\text { miRVana } \\
\text { PARIS kit } \\
\text { (Ambion) }\end{array}$ & $\begin{array}{l}\text { miR-372- } \\
3 p(n=1) \\
\text { miR-367- } \\
3 p(n=1)\end{array}$ & No & No & $\begin{array}{c}\text { Small RNA } \\
\text { concentration }\end{array}$ & $\begin{array}{c}\text { Suggested } \\
\text { additional } \\
\text { specificity of } \\
\text { using } \\
\text { miR-367-3p }\end{array}$ \\
\hline $\begin{array}{l}\text { Dieckmann } \\
\text { et al. (2012) } \\
{ }_{13}\end{array}$ & 200 & $\begin{array}{l}\text { miRNeasy } \\
\text { mini kit } \\
\text { (Qiagen) }\end{array}$ & $\begin{array}{c}\mathrm{miR}- \\
371 \sim 373 \\
(\mathrm{n}=3)\end{array}$ & No & No & 18S RNA & $\begin{array}{c}\text { Different reverse } \\
\text { transcription } \\
\text { step for } 18 \mathrm{~S} \\
\text { versus } \\
\text { microRNAs, } \\
\text { risking technical } \\
\text { bias } \\
\end{array}$ \\
\hline $\begin{array}{l}\text { Gillis et al. } \\
(2013)^{66}\end{array}$ & 50 & $\begin{array}{c}\text { TaqMan } \\
\text { miRNA ABC } \\
\text { purification } \\
\text { kit; Panel A } \\
\text { (Life } \\
\text { Technologies) }\end{array}$ & $\begin{array}{c}\text { miR- } \\
371 \sim 373 \\
(\mathrm{n}=3) \\
\text { miR- } \\
302 / 367 \\
(\mathrm{n}=4)\end{array}$ & No & $\begin{array}{c}\text { Yes; } \\
\text { cel-miR- } \\
\text { 39-3p } \\
\text { ath-miR- } \\
\text { 159a }\end{array}$ & $\begin{array}{l}\text { miR-20a-5p } \\
\text { miR-93-5p }\end{array}$ & $\begin{array}{c}\text { Four serum } \\
\text { microRNA } \\
\text { panel* identified } \\
\text { as most sensitive } \\
\text { and specific }\end{array}$ \\
\hline $\begin{array}{l}\text { Syring et al. } \\
(2015)^{70}\end{array}$ & 400 & $\begin{array}{l}\text { miRVana } \\
\text { PARIS kit } \\
\text { (Ambion) }\end{array}$ & $\begin{array}{c}\text { miR- } \\
371 \sim 373 \\
(\mathrm{n}=3) \\
\text { miR- } \\
302 / 367 \\
(\mathrm{n}=4)\end{array}$ & No & $\begin{array}{c}\text { Yes; } \\
\text { cel-miR- } \\
39-3 p\end{array}$ & None & $\begin{array}{c}\text { Four serum } \\
\text { microRNA } \\
\text { panel* } \\
\text { confirmed as } \\
\text { most sensitive } \\
\text { and specific }\end{array}$ \\
\hline $\begin{array}{l}\text { Spiekermann } \\
\text { et al. } \\
(2015)^{68}\end{array}$ & 200 & $\begin{array}{l}\text { miRNeasy } \\
\text { mini kit } \\
\text { (Qiagen) }\end{array}$ & $\begin{array}{c}\mathrm{miR}-371 \mathrm{a}- \\
3 \mathrm{p}(\mathrm{n}=1)\end{array}$ & No & No & miR-20a-5p & $\begin{array}{c}\text { Only single } \\
\text { microRNA } \\
\text { tested }\end{array}$ \\
\hline $\begin{array}{l}\text { Rijlaarsdam } \\
\text { et al. (2015) } \\
\quad 67\end{array}$ & 50 & $\begin{array}{c}\text { TaqMan } \\
\text { miRNA ABC } \\
\text { purification } \\
\text { kit; Panels } \\
\text { A\&B } \\
\text { (Life } \\
\text { Technologies) }\end{array}$ & $\begin{array}{l}\text { Global } \\
\text { profiling } \\
\text { study } \\
(\mathrm{n} \sim 750)\end{array}$ & No & $\begin{array}{c}\text { Yes; } \\
\text { ath-miR- } \\
159 a\end{array}$ & $\begin{array}{c}\text { Global } \\
\text { normalisation } \\
\text { (PMID } \\
19531210)\end{array}$ & $\begin{array}{c}\text { Appropriate } \\
\text { normalization } \\
\text { approach for a } \\
\text { global profiling } \\
\text { study used } \\
\text { Confirmation of } \\
\text { the relevance of } \\
\text { miR-371-373 } \\
\text { microRNAs. }\end{array}$ \\
\hline $\begin{array}{l}\text { Spiekermann } \\
\text { et al. (2015) } \\
69\end{array}$ & 200 & $\begin{array}{l}\text { miRNeasy } \\
\text { mini kit } \\
\text { (Qiagen) }\end{array}$ & $\begin{array}{c}\mathrm{miR}-371- \\
373(\mathrm{n}=3)\end{array}$ & No & No & $\begin{array}{l}\text { 18S RNA, but } \\
\text { suggested } \\
\text { none required }\end{array}$ & $\begin{array}{l}\text { Different reverse } \\
\text { transcription } \\
\text { step for } 18 \mathrm{~S} \text {. } \\
\text { Suggestion that }\end{array}$ \\
\hline
\end{tabular}




\begin{tabular}{|c|c|c|c|c|c|c|c|}
\hline & & & & & & $\begin{array}{c}\text { normalization ( } \\
\text { relative } \\
\text { quantification) } \\
\text { not required }\end{array}$ \\
\hline $\begin{array}{c}\text { Murray et al. } \\
(2016)^{12}\end{array}$ & 200 & $\begin{array}{c}\text { miRNeasy } \\
\text { serum/plasma } \\
\text { kit (Qiagen) }\end{array}$ & $\begin{array}{c}\text { miR-371- } \\
373(\mathrm{n}=3) \\
\text { miR-302- } \\
367(\mathrm{n}=5)\end{array}$ & $\begin{array}{c}\text { Yes: } \\
\Delta \mathrm{Ct}(\mathrm{miR}- \\
23 \mathrm{a}-3 \mathrm{p}- \\
\text { miR-451a) }\end{array}$ & $\begin{array}{c}\text { Yes: } \\
\text { cel-miR- } \\
39-3 \mathrm{p}\end{array}$ & miR-30b-5p & $\begin{array}{c}\text { Four serum } \\
\text { microRNA } \\
\text { panel* } \\
\text { confirmed. } \\
\text { First } \\
\text { demonstration of } \\
\text { relapse detection }\end{array}$ \\
\hline
\end{tabular}

* Four serum microRNA panel comprising miR-371a-3p, miR-372-3p, miR-373-3p and miR-367-3p 